LBL-35432

UC-401

\title{
A Classical Model for Closed-Loop Diagrams of Binary Liquid Mixtures
}

\author{
J. v. Schnitzler and J. M. Prousnitz \\ Department of Chemical Engineering \\ University of California \\ and \\ Chemical Sciences Division \\ Lawrence Berkeley Laboratory \\ University of California \\ Berkeley, CA 94720, U.S.A.
}

\section{March 1994}

This work was supported by the Director, Office of Energy Research, Office of Basic Energy Sciences, Chemical Sciences Division of the U.S. Department of Energy under Contract Number DE-AC03-76SF00098.

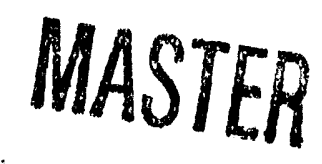




\section{A Classical Model for Closed-Loop Diagrams of Binary Liquid Mixtures}

J. v. Schnitzler and J.M. Prausnitz

Department of Chemical Engineering

University of California

Berkeley

March 1994 


\section{Abstract}

A classical lattice model for closed-loop temperature-composition phase diagrams has been developed. It considers the effect of specific interactions, such as hydrogen bonding, between dissimilar components. This van Laar-type model includes a Flory-Huggins term for the excess entropy of mixing. It is applied to several liquid-liquid equilibria of nonelectrolytes, where the molecules of the two components differ in size.

The model is able to represent the observed data semi-quantitatively, but in most cases it is not flexible enough to predict all parts of the closed loop quantitatively. The ability of the model to represent different binary systems is discussed. Finally, attention is given to a correction term, concerning the effect of concentration fluctuations near the upper critical solution temperature.

\section{Introduction}

Partially miscible binary liquid systems occur frequently in nature. In most cases mutual miscibility rises with temperature; at some upper critical solution temperature, partial immiscibility disappears and the binary system becomes completely miscible for all compositions. In some cases, however, immiscibility rises with temperature; these systems exhibit a lower critical solution temperature such that complete miscibility is observed below that temperature. Finally, there are some binary systems which exhibit both upper and lower critical solution temperatures. Here we must distinguish between two cases: in the first case, the lower critical solution temperature lies above the upper critical solution temperature while in the second case, the lower critical solution temperature lies below the upper critical solution temperature. It is this second case which gives closed-loop diagrams as shown in Figure 1.

A simple classical model, discussed here, can be used to describe a closed-loop diagram. This model is classical in the sense that it is not based on the modern theory of fluids very close to the critical region. Because of this classical feature, 
the model is able to give only a reasonable but not totally satisfactory fit of experimental data.

Before presenting the model, it is useful to consider the physical factors which determine the binary phase diagram, i.e. a plot of temperature versus composition at an ordinary constant pressure.

Binary mixtures of typical nonpolar liquids show positive deviations from Raoult's law due to an endothermic enthalpy of mixing. When that enthalpy is appreciably larger than the thermal energy (RT), the liquid mixture is unstable or metastable for a range of compositions, giving two liquid phases. As the temperature rises, stability is favored such that there is no tendency to split into two phases at temperatures exeeding the upper critical solution temperature.

When a binary liquid mixture contains components that can interact strongly with one another, deviations from Raoult's law are negative or weakly positive. In that event, there is no instability and no tendency to form two liquid phases. However, the effect of the strong interaction between unlike components is often temperature-dependent such that the effect of strong interaction becomes weaker as the temperature rises; a common example is provided by hydrogen bonding where the strength of the hydrogen bond is (nearly) independent of temperature but where the number of hydrogen-bonded pairs falls as the temperature rises.

Hydrogen bonding is exothermic. As the temperature rises and as hydrogen bonds between dissimilar molecules are broken, the enthalpy oi mixing becomes increasingly endothermic. At a sufficiently high temperature, when the endothermic enthalpy has become so large that it is significantly in excess of the thermal energy RT, the liquid mixture becomes unstable for a range of compositions and two liquid phases form. Further increases in temperature make the enthalpy of mixing more endothermic, raising the range of unstable compositions up to some maximum. At some temperature, further increases in temperature no longer change the enthalpy of mixing, and therefore increases in temperature lower the ratio of enthalpy of mixing to kinetic energy. The range of immiscibility decreases until, at some high temperature, the system becomes completely miscible in all proportions. 
We must now briefly consider the other case, when the phase diagram contains both a lower critical solution temperature and an upper critical solution temperature, such that the lower critical solution temperature is above the upper critical solution temperature. The physical reason for the lower critical solution temperature now follows not from considerations of strong exothermic interactions between unlike molecules but, instead, from free-volume effects: if liquid component 1 is near its (vapor-liquid) critical temperature while liquid component 2 is not, mixing at constant temperature and pressure produces an appreciable negative volume change. This negative change affects the entropy of mixing such that the liquid mixture becomes unstable for a range of compositions which rises as the temperature increases. In this work, we do not consider such mixtures.

When we mix two fluids 1 and 2, whose molecules can form a strong 1-2 interaction, there is a competition between the "normal" endothermic enthalpy of interaction between two different molecules and the "oriented" (or specific) interaction that produces an exothermic enthalpy of interaction. This competition is temperature dependent; it depends on the Boltzmann factor as discussed by ten Brinke and Karasz [1] and as indicated in the next section.

\subsection{Closed-Loop Systems: Symmetric Mixtures}

Consider a binary mixture containing $\mathrm{N}_{1}$ molecules of component 1 and $\mathrm{N}_{2}$ molecules of component 2 where the molecular size and shape of 1 are nearly the same as those of 2 . We place these molecules on a three-dimensional lattice where each molecule occupies one lattice point. There are no holes in this lattice; the total number of lattice points is $\mathrm{N}_{1}+\mathrm{N}_{2}$.

Assuming that $U$, the total potential energy of this system, is pair-wise additive

$$
\mathrm{U}=\frac{\mathrm{z}}{2}\left(\mathrm{~N}_{1}+\mathrm{N}_{2}\right)\left[\mathrm{x}_{1}^{2} \varepsilon_{11}+\mathrm{x}_{2}^{2} \varepsilon_{22}+2 \mathrm{x}_{1} \mathrm{x}_{2}(1-f) \varepsilon_{12}+2 \mathrm{x}_{1} \mathrm{x}_{2} f \varepsilon_{12}^{\prime}\right]
$$


where the mole fraction $x_{i}=N_{i} /\left(N_{1}+N_{2}\right)$

and $\mathrm{z}$ is the coordination number. The characteristic energy for a $1-1$ pair is $\varepsilon_{11}$ and that for a 2-2 pair is $\varepsilon_{22}$. For 1-2 pairs, we distinguish between "normal" interactions characterized by $\varepsilon_{12}$ and "oriented" interactions characterized by $\varepsilon_{12}^{\prime}$. Here "normal' refers to nonpolar dispersion-force interactions while "oriented" refers to specific interactions such as hydrogen bonds or charge-transfer interactions. The fraction of 1-2 interactions which is "normal" is designated by $1-f$ and that which is "oriented" is $f$.

The energy of mixing (here designated as the excess energy UE) is given by

$$
\mathrm{U}^{\mathrm{E}}=\mathrm{U}-\frac{\mathrm{z}}{2}\left(\mathrm{~N}_{1}+\mathrm{N}_{2}\right)\left(\mathrm{x}_{1}^{2} \varepsilon_{11}+\mathrm{x}_{2}^{2} \varepsilon_{22}\right)
$$

The ratio (1-f)/f is given by Boltzmann statistics:

$$
\frac{1-\mathrm{f}}{\mathrm{f}}=\omega \cdot \exp \left[\frac{\delta \varepsilon_{12}}{\mathrm{kT}}\right]
$$

where $\delta \varepsilon_{12}=\varepsilon_{12}^{\prime}-\varepsilon_{12}$ and $\omega$ is an entropic degeneracy factor, the ratio of the degeneracy of "normal" interactions to that of "oriented" interactions. Eq. (2.3) is rewritten in the form

$$
f=\left(\omega \exp \left[\frac{\delta \varepsilon_{12}}{\mathrm{kT}}\right]+1\right)^{-1}
$$

Substitution in Eq. (2.2) gives

$$
\begin{aligned}
& \mathrm{U}^{\mathrm{E}}=\mathrm{z}\left(\mathrm{N}_{1}+\mathrm{N}_{2}\right) \mathrm{x}_{1} \mathrm{x}_{2}\left[\Delta \varepsilon_{12}+\delta \varepsilon_{12}\left(\omega \exp \left[\frac{\delta \varepsilon_{12}}{\mathrm{kT}}\right]+1\right)^{-1}\right] \\
& \text { where } \Delta \varepsilon_{12}=\varepsilon_{12}-\frac{1}{2}\left(\varepsilon_{11}+\varepsilon_{22}\right)
\end{aligned}
$$


For a "normal" liquid mixture of nonelectrolytes, there is no orientation ( $f=0$; $\left.\omega=1 ; \delta \varepsilon_{12}=0\right)$ and $\Delta \varepsilon_{12}$ is positive because, typically $\left|\varepsilon_{12}\right|<\left|\left(\varepsilon_{11} \varepsilon_{22}\right)^{1 / 2}\right|$.

The molar excess energy is $u^{E}=\frac{U^{E}}{N_{1}+N_{2}} \cdot N_{A v}$

where $\mathrm{N}_{\mathrm{Av}}$ is Avogrado's number.

The molar excess Helmholtz energy $\mathrm{a}^{\mathrm{E}}$ is related to $\mathrm{u}^{\mathrm{E}}$ by the Gibbs-Helmholtz equation

$$
\left(\frac{\partial \mathrm{a}^{\mathrm{E}} / \mathrm{T}}{\partial 1 / \mathrm{T}}\right)_{\mathrm{v}, \mathrm{x}}=\mathrm{u}^{\mathrm{E}}
$$

Integrating Eq. (2.6), between the limits $1 / \mathrm{T}=0$ and $1 / \mathrm{T}$, at constant volume $\mathrm{V}$ and composition $\mathrm{x}$, gives:

$$
\frac{a^{E}}{R T}=z x_{1} x_{2} \cdot \frac{B}{R T}+\left(\frac{a^{E}}{R T}\right)_{\frac{1}{T} \rightarrow 0}
$$

where

$$
\mathrm{B}=\Delta \varepsilon_{12}+\delta \varepsilon_{12}-\mathrm{RT} \cdot \ln \left[\frac{\omega \exp \left(\delta \varepsilon_{12} / \mathrm{RT}\right)+1}{\omega+1}\right]
$$

where the energies $\Delta \varepsilon_{12}$ and $\delta \varepsilon_{12}$ are now in molar units.

At very high temperature, the kinetic energy becomes much larger than the potential energy and therefore the ratio $u^{E} / R T$ goes to zero. That reduces the excess Helmholtz energy to an entropic term, $-\mathrm{s}^{\mathrm{E}} / \mathrm{R}$, the excess entropy of mixing:

$$
\left(\frac{a^{E}}{R T}\right)_{\frac{1}{T} \rightarrow 0}=\frac{-s^{E}}{R}
$$


In the present work we use a Flory - Huggins term for the athermal excess entropy of mixing

$$
\frac{-s^{E}}{R}=\left(x_{1} \ln \frac{\varphi_{1}}{x_{1}}+x_{2} \ln \frac{\varphi_{2}}{x_{2}}\right)
$$

where $\varphi_{i}$ is the volume fraction of component $i$. For a symmetric system (equisized molecules), $\varphi_{i}=x_{i}$ and therefore $\mathrm{s}^{\mathrm{E}}=0$.

As shown by Scott [2], at low pressure, we can use the excellent approximation

$$
\left(\mathrm{a}^{\mathrm{E}}\right)_{\mathrm{T}, \mathrm{V}}=\left(\mathrm{g}^{\mathrm{E}}\right)_{\mathrm{T}, \mathrm{P}}
$$

where $\mathrm{gE}^{\mathrm{E}}$ is the excess molar Gibbs energy.

For mixtures of equisized molecules, Eqs. (2.7) to (2.11) give:

$$
\frac{\mathrm{g}^{\mathrm{E}}}{\mathrm{RT}}=\mathrm{zx}_{1} \mathrm{x}_{2} \cdot \frac{\mathrm{B}}{\mathrm{RT}}
$$

The activity coefficients $\gamma$ are found from

$$
\mathrm{RT} \cdot \ln \gamma_{\mathrm{i}}=\left(\frac{\partial\left(\mathrm{n}_{\mathrm{t}} \cdot \mathrm{g}^{\mathrm{E}}\right)}{\partial \mathrm{n}_{\mathrm{i}}}\right)_{\mathrm{T}, \mathrm{P}, \mathrm{n}_{\mathbf{j} \neq \mathrm{i}}}
$$

where $n_{i}$ is the number of moles of component $i$ and $n_{t}=n_{1}+n_{2}$. Liquid-liquid equilibria are calculated from

$$
\begin{aligned}
& \left(\gamma_{1} \mathrm{x}_{1}\right)^{\prime}=\left(\gamma_{1} \mathrm{x}_{1}\right)^{\prime \prime} \\
& \left(\gamma_{2} \mathrm{x}_{2}\right)^{\prime}=\left(\gamma_{2} \mathrm{x}_{2}\right)^{\prime \prime}
\end{aligned}
$$

and 


$$
\left(\mathrm{x}_{1}+\mathrm{x}_{2}\right)^{\prime}=1 \quad \text { and } \quad\left(\mathrm{x}_{1}+\mathrm{x}_{2}\right)^{\prime \prime}=1
$$

where ()' and ()" designate the two liquid phases at equilibrium.

\subsection{Closed-Loop Systems: Unsymmetric Mixtures}

When the size and shape of molecule 1 are significantly different from those of molecule 2, we generalize the lattice model using the method of van Laar as discussed by Wohl [3]. We then obtain

$$
\frac{g^{E}}{R T}=\frac{\mathrm{zx}_{1} \mathrm{x}_{2} \mathrm{q}_{1} \mathrm{q}_{2}}{\left(\mathrm{x}_{1} \mathrm{q}_{1}+\mathrm{x}_{2} \mathrm{q}_{2}\right)} \cdot \frac{\mathrm{B}}{\mathrm{RT}}+\mathrm{x}_{1} \ln \frac{\varphi_{1}}{\mathrm{x}_{1}}+\mathrm{x}_{2} \ln \frac{\varphi_{2}}{\mathrm{x}_{2}}
$$

where $q_{i}$ is a size parameter for molecule $i$ and $\varphi_{i}$ is again the volume fraction. $\mathrm{B}$, as before, is given by Eq. (2.8).

By introducing the van Laar method we require an additional parameter $q_{2}$, where 2 designates the larger molecule. Without loss of generality, we set $\mathrm{q}_{1}=1$. Further, we assume the ratio of the size parameters to be approximately the same as the ratio of the molar volumes. Therefore, $\varphi_{i}$ can be defined as

$$
\varphi_{1}=\frac{\mathrm{x}_{1}}{\mathrm{x}_{1}+\mathrm{q}_{2} \cdot \mathrm{x}_{2}} \quad \text { and } \quad \varphi_{2}=\frac{\mathrm{q}_{2} \cdot \mathrm{x}_{2}}{\mathrm{x}_{1}+\mathrm{q}_{2} \cdot \mathrm{x}_{2}}
$$

As before, activity coefficients are found from Eq. (2.13).

$$
\ln \gamma_{1}=\frac{\mathrm{z}}{\left(\frac{\mathrm{x}_{1}}{\mathrm{x}_{2} \mathrm{q}_{2}}+1\right)^{2}} \cdot \frac{\mathrm{B}}{\mathrm{RT}}+\ln \left(\frac{\varphi_{1}}{\mathrm{x}_{1}}\right)+\left(1-\frac{\varphi_{1}}{\mathrm{x}_{1}}\right)
$$




$$
\ln \gamma_{2}=\frac{\mathrm{z} \cdot \mathrm{q}_{2}}{\left(\frac{\mathrm{x}_{2} \mathrm{q}_{2}}{\mathrm{x}_{1}}+1\right)^{2}} \cdot \frac{\mathrm{B}}{\mathrm{RT}}+\ln \left(\frac{\varphi_{2}}{\mathrm{x}_{2}}\right)+\left(1-\frac{\varphi_{2}}{\mathrm{x}_{2}}\right)
$$

Liquid-liquid equilibria are again found from Equations (2.14) and (2.15).

Equations (2.18) are the well-known van Laar equations with a Flory-Huggins correction for size asymmetry. The important new feature of Eqs (2.18) is the temperature dependence for constant B, given by Equation (2.8).

\section{Results}

To fit experimental data to the van Laar-Flory-Huggins model, we first estimate size parameter $\mathrm{q}_{2}$ from the ratio the UNIQUAC pure-component parameters ( $R$ and $\mathrm{Q})$. To optimize agreement with experiment, $\mathrm{q}_{2}$ is allowed to be slightly adjusted. Table 1 shows parameters $\mathrm{q}_{2}$ for the systems chosen in this work, compared to the two UNIQUAC parameters. In general $\mathrm{q}_{2}$ lies close to the ratio of the UNIQUAC surface-parameters $Q_{2} / Q_{1}$, or between this value and the ratio of the UNIQUAC volume- parameters $R_{2} / R_{1}$.

Having selected $q_{2}$, we must then find parameters $\Delta \varepsilon_{12}, \delta \varepsilon_{12}$ and $\omega$ from binary data. From experience we know that $\Delta \varepsilon_{12}$ is positive; a reasonable value for $\Delta \varepsilon_{12}$ (in molar units) is in the region RT, perhaps $100-1000 \mathrm{cal} / \mathrm{mol}$. Parameter $\delta \varepsilon_{12}$ is negative and in the region $1000-10,000 \mathrm{cal} / \mathrm{mol}$. For systems where molecule 1 can hydrogen bond with molecule $2, \omega$ is a positive number much larger than unity.

Figures 2 to 9 show comparisons of calculated and observed phase diagrams. Figures 4,5 and 6 show the influence of parameters $q_{2}, \Delta \varepsilon_{12}$ and $\delta \varepsilon_{12}$ of the model. Figure 3, for Glycerol/Benzyl-Ethylamine, gives two sets of $\Delta \varepsilon_{12} / \delta \varepsilon_{12}$, which change the UCST. Figure 4 shows the influence of $q_{2}$; by increasing $q_{2}$, the closed loop shifts in the direction of pure component $1\left(\mathrm{x}_{2}=0\right)$ and decreases in 
width at constant critical solution temperatures. Further, a rise in $\omega$ increases the width of the closed loop.

These examples indicate that Eq. (2.16) can give a fair representation of the experimental data but, in typical cases, when the loop is forced to go thro'igh the two observed critical solution temperatures, it is too narrow. Clearly, the simple model presented here has some deficiencies.

In the region near the LCST, the fit is much better than that in the region near the UCST. This result is not surprising because this model is based on specific interactions. These specific interactions are important for the LCST, but at higher temperature, in the region of the UCST, the specific interactions, such as hydrogen bonding, are not important. In other words, intermolecular forces responsible for UCST are different from those that cause the LCST; they are not seriously considered in the present lattice model. For example, at fixed composition, the number of 1-2 pairs, $\mathrm{N}_{12}$, is assumed to be constant, over the temperature range of the closed loops. Other investigations [4] have been made, where $\mathrm{N}_{12}$ is given as a function of temperature. Those models include a nonrandomness factor.

Figure 8 compares results of a nonrandom model, as discussed by T. Hino et.al. [4], with those obtained by using the model discused in this work. The nonrandom model is able to provide a closed loop, wider in composition range (at fixed critical Temperatures, UCST and LCST) than that obtained from the randommixing model.

If we compare the results of the different systems, it appears that the systems where UCST and LCST are close together allow the better fit, especially near the UCST. A good example is provided by the system Water/1-Propoxy-2-Propanol, where the difference between UCST and LCST is less then $140 \mathrm{~K}$; for the system Glycerol/Benzyl-Ethylamine, immiscibility extends over $220 \mathrm{~K}$. The larger the temperature difference between UCST and LCST, the more important become factors not considered in the model presented here.

Finally, consider the effect of concentration fluctuations in the critical region. J.J. de Pablo and J.M. Prausnitz [5] discuss a correction term considering fluctuations 
near, or at the UCST. The idea of this correction term is to introduce fluctuations through appropriate composition averages. The instantaneous mole fraction $x_{i}$ is related to the time-average mole fraction $\bar{x}_{i}$ through

$$
\mathrm{x}_{\mathrm{i}}=\overline{\mathrm{x}}_{\mathrm{i}}+\delta \mathrm{x}_{\mathrm{i}}
$$

where $\delta x_{i}$ is a fluctuation. To take into account the effect of fluctuation on the excess Gibbs energy; i.e. for a simple two suffix Margules equation, we now write

$$
\mathrm{g}^{\mathrm{E}}=\mathrm{B} \cdot \overline{\mathrm{x}_{1} \mathrm{x}_{2}}=\mathrm{B} \cdot\left(\overline{\mathrm{x}}_{1} \overline{\mathrm{x}}_{2}+\overline{\delta \mathrm{x}_{1} \delta \mathrm{x}_{2}}\right)
$$

where the overbars denote time average. Note that whereas $\bar{\delta} \bar{x}_{1}=0$ and $\overline{\delta \mathrm{x}_{2}}=0$, the product $\overline{\delta \mathrm{x}_{1} \delta \mathrm{x}_{2}} \neq 0$.

To introduce the effect of fluctuation on the excess Gibbs energy, de Pablo proposed a reasonable but essentially empirical fluctuation-correction term. This fluctuation-correction is significient in the critical region, but it becomes negligible remote from the critical region. The fluctuation term depends on the distance from the critical point. This method does not require any additional adjustable parameters, but it does require knoweledge of the coordinates of the critical point $\left(\mathrm{T}_{\mathrm{c}}=\mathrm{UCST}\right.$ and critical composition $\left.\mathrm{x}_{1 \mathrm{c}}\right)$.

Combining the fluctuation term from de Pablo with the van Laar-model discussed in this work, the excess Gibbs energy (Eq.2.16) can be written as

$$
\frac{g^{E}}{R T}=\frac{z x_{1} x_{2} q_{1} q_{2}}{\left(x_{1} q_{1}+x_{2} q_{2}\right)} \cdot \frac{B}{R T} \cdot\left(1-K \cdot \exp \left[a \cdot D^{b}\right]\right)+x_{1} \ln \frac{\varphi_{1}}{x_{1}}+x_{2} \ln \frac{\varphi_{2}}{x_{2}}
$$

where $\mathrm{K}$ can be calculated from $\mathrm{T}_{\mathrm{c}}, \mathrm{x}_{1 \mathrm{c}}$ and $\mathrm{B}$. In contrast to de Pablo's work, $\mathrm{K}$, in Eq. (3.2), depends on the temperature, as B is a function of the temperature. D is the distance of the temperature $T$ to the critical temperature $T_{c}=U C S T$. Parameters $a$ and $b$ are determined from critical exponents $\alpha$ and $\beta$ as discussed by de Pablo[5]. These critical exponents are part of the equations that describe the 
shape of the coexistence curve close to the critical point, as discussed by Greer and Moldover [6], and more recently by Ewing et.al. [7]. To show the effect of the fluctuation term, the van Laar-fluctuation model (Eq. 3.2) is applied to the system Glycerol / Benzyl-Ethylamine, shown in Figure 9. Parameters a and b are found to be $a=-18$ and $b=0.36$.

The main effect of the fluctuation term is to flatten the coexistence loop in the region of UCST. The van Laar-fluctuation model (Eq. 3.2) provides a better fitting ability than the 'normal' van Laar-model. Although, the fluctuation term only requires knowledge of the critical coordinates, the van Laar-fluctuation model provides an excellent fit of the experimental closed-loop data.

\section{Conclusion}

To calculate closed-loop phase diagrams, a classical lattice model for binary liquid-liquid mixtures has been discussed. It is a model based on the van Laar equation for the excess Gibbs energy, including a temperature-dependent term to take into account specific interactions between dissimilar molecules.

The model is able to give fair results for most of the systems but, particularly near the UCST, it has its limits. By adding a fluctuation correction term, as discussed by de Pablo for systems with an UCST, the ability of the model to represent the data improves significiantly, without requiring any additional adjustable parameters. 


\section{Tables}

\begin{tabular}{|l|c|c|c|}
\hline System & optimized q2 & $\begin{array}{c}\text { UNIQUAC } \\
\mathrm{Q}_{2} / \mathrm{Q}_{1}\end{array}$ & $\begin{array}{c}\text { UNIQUAC } \\
\mathrm{R}_{2} / \mathrm{R}_{1}\end{array}$ \\
\hline Water/1-Propoxy-2-Propanol & 4.20 & 3.12 & 5.48 \\
\hline Water/2-Propoxy-1-Propanol & 4.20 & 3.12 & 5.48 \\
\hline Water/Nicotine & 5.00 & 3.30 & 7.05 \\
\hline Glycerol/Benzyl-Ethylamine & 1.40 & 1.45 & 1.62 \\
\hline Glycerol/m-Toluidine & 1.11 & 1.11 & 1.24 \\
\hline
\end{tabular}

Table 1: Comparison of parameter $\mathrm{q}_{2}$ chosen in this work, and the UNIQUAC parameters

\begin{tabular}{|l|c|c|c|}
\hline System & $\begin{array}{c}\Delta \varepsilon_{12} \\
{[\mathrm{cal} / \mathrm{mol}]}\end{array}$ & $\begin{array}{c}\delta \varepsilon_{12} \\
{[\mathrm{cal} / \mathrm{mol}]}\end{array}$ & $\omega$ \\
\hline Water/1-Propoxy-2-Propanol & 177 & -4230 & 6000 \\
\hline Water/2-Propoxy-1-Propanol & 178 & -4010 & 6000 \\
\hline Water/Nicotine & 185 & -4150 & 5000 \\
\hline Glycerol/Benzyl-Ethylamine & 332 & -5000 & 10000 \\
\hline Glycerol/m-Toluidine & 275 & -3900 & 5000 \\
\hline
\end{tabular}

Table 2: Parameters for the calculated closed loops 


\section{Figures}

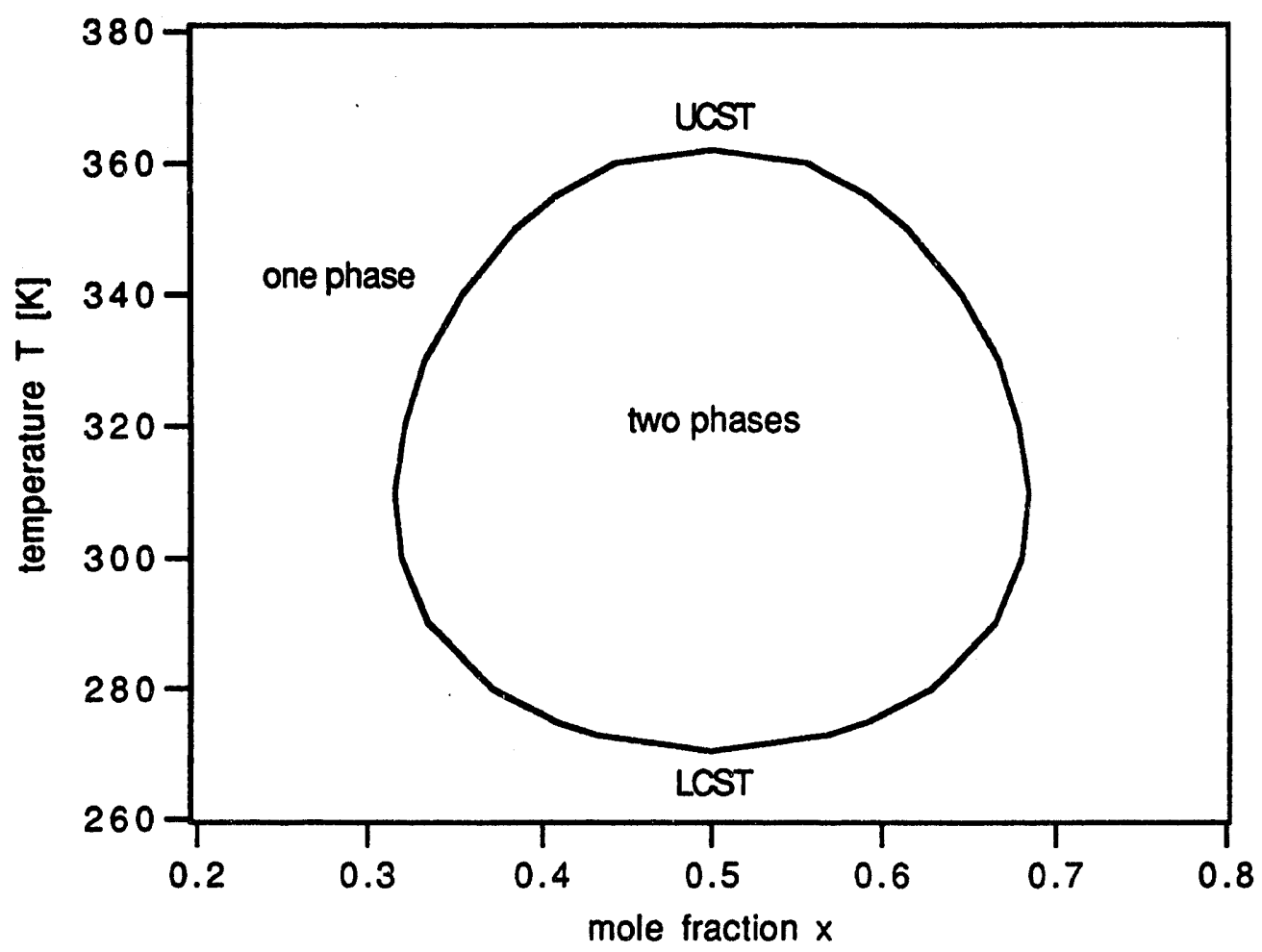

Figure 1: General Closed-Loop Diagram

(UCST = upper critical solution temperature, LCST = lower critical solution temperature) 


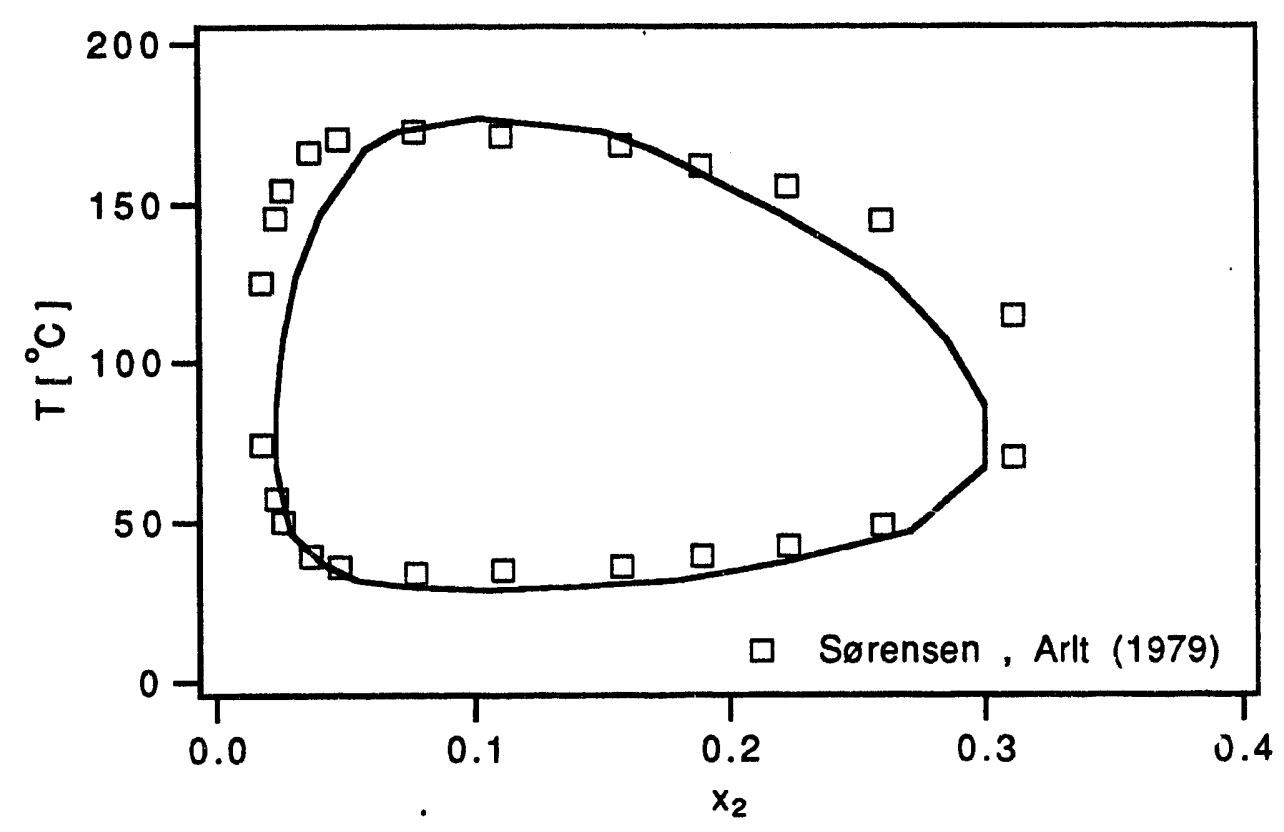

Figure 2: Closed-Loop Diagram for Water (1) / 1-Propoxy-2-Propanol (2) Parameter: $\Delta \varepsilon 12=177[\mathrm{cal} / \mathrm{mol}], \delta \varepsilon 12=-4230[\mathrm{cal} / \mathrm{mol}], \omega=6000, \mathrm{q} 2=4.2$

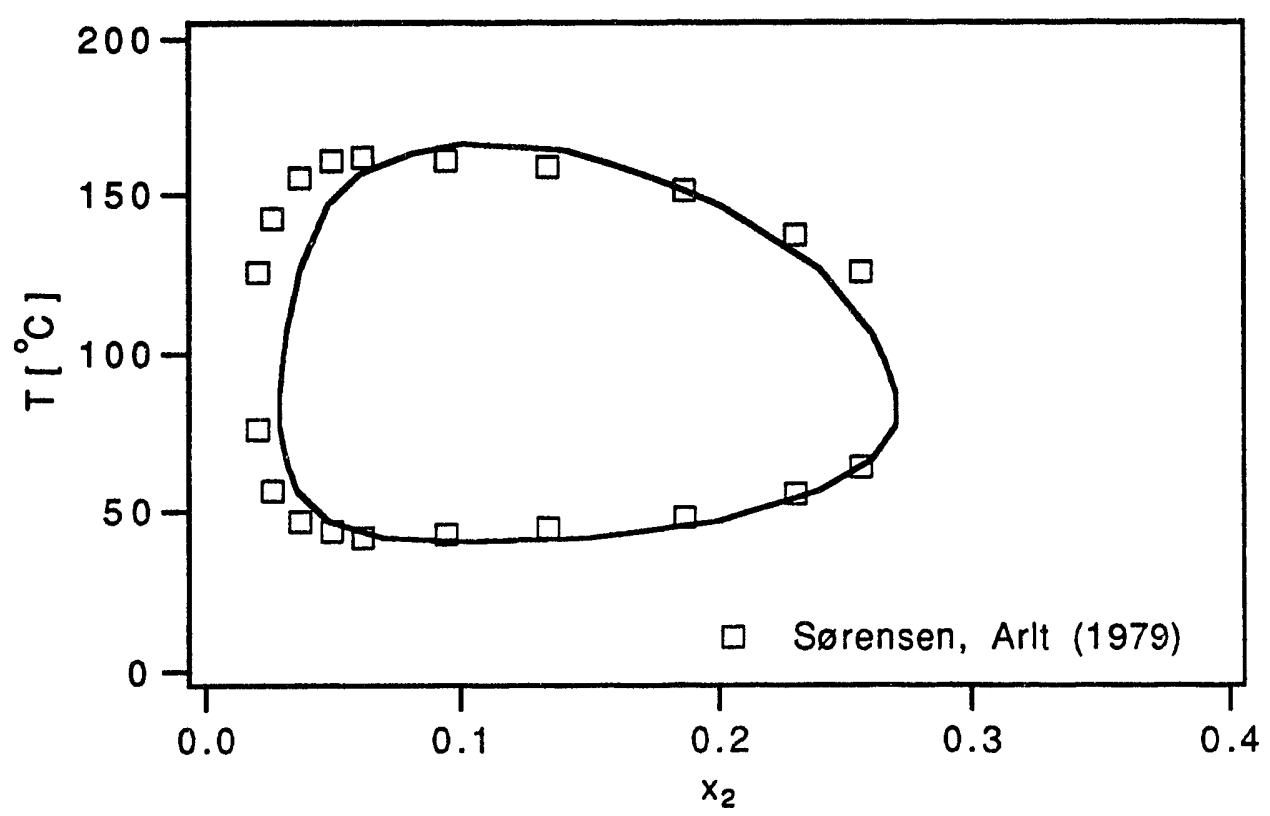

Figure 3: Closed-Loop Diagram for Water (1) / 2-Propoxy-1Propanol (2) Parameter: $\Delta \varepsilon_{12}=178[\mathrm{cal} / \mathrm{mol}], \delta \varepsilon_{12}=-4010[\mathrm{cal} / \mathrm{mol}], \omega=6000, \mathrm{q} 2=4.2$ 


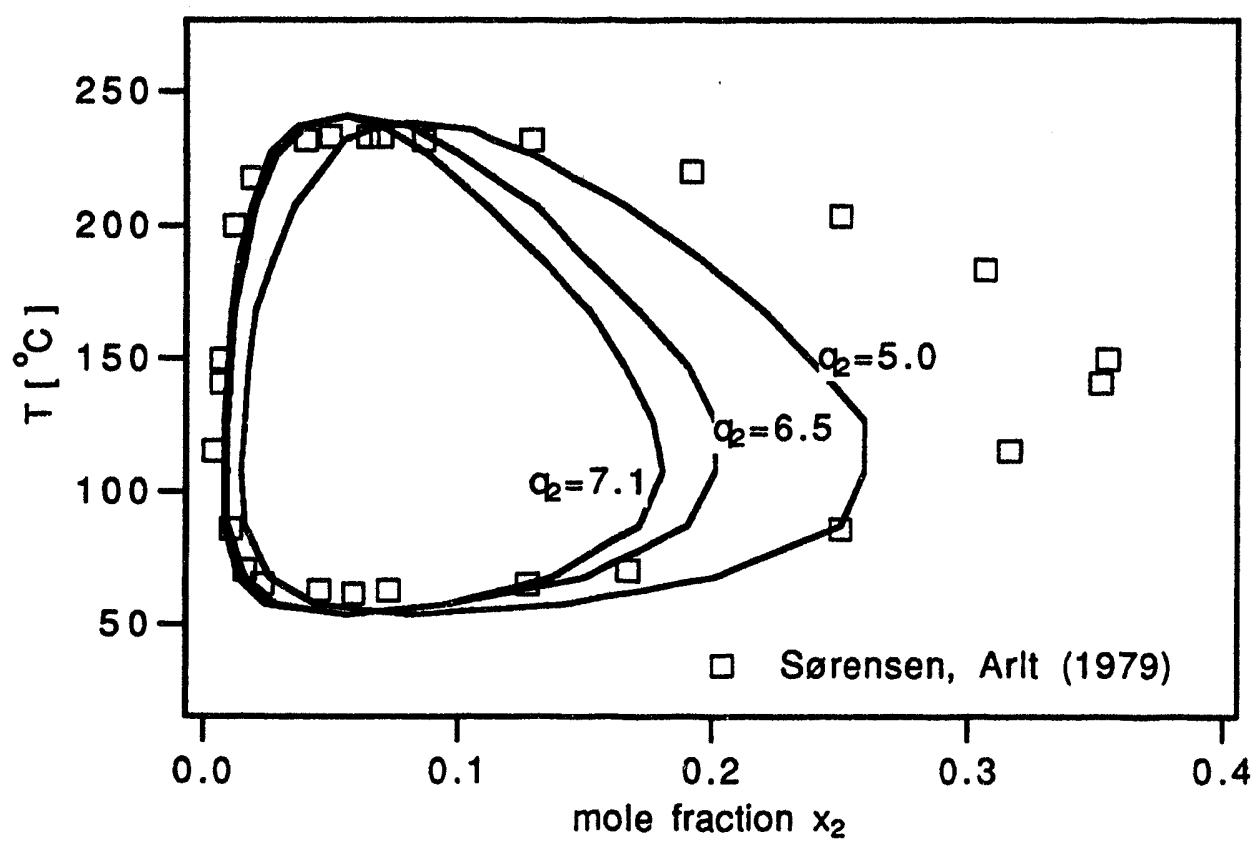

Figure 4: Closed-Loop Diagram for Water (1)/ Nicotine (2)

Parameter: $\Delta \varepsilon_{12}=185$ [cal $\left./ \mathrm{mol}\right], \delta \varepsilon_{12}=-4150[\mathrm{cal} / \mathrm{mol}], \omega=5000$

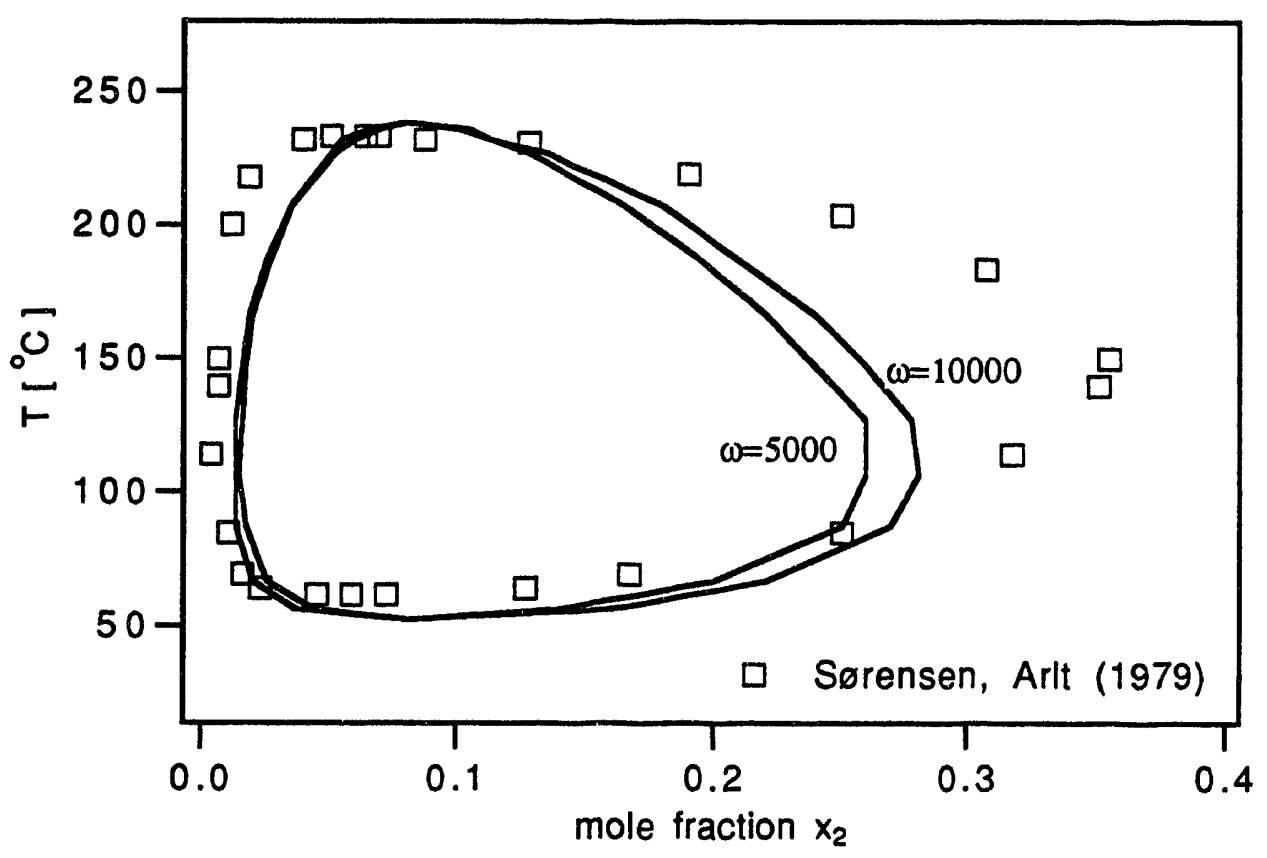


Figure 5: Closed-Loop Diagram for Water (1)/ Nicotine (2)

Parameter: $\Delta \varepsilon_{12}=185[\mathrm{cal} / \mathrm{mol}], \delta \varepsilon_{12}=-4150[\mathrm{cal} / \mathrm{mol}], \mathrm{q}_{2}=5.0$

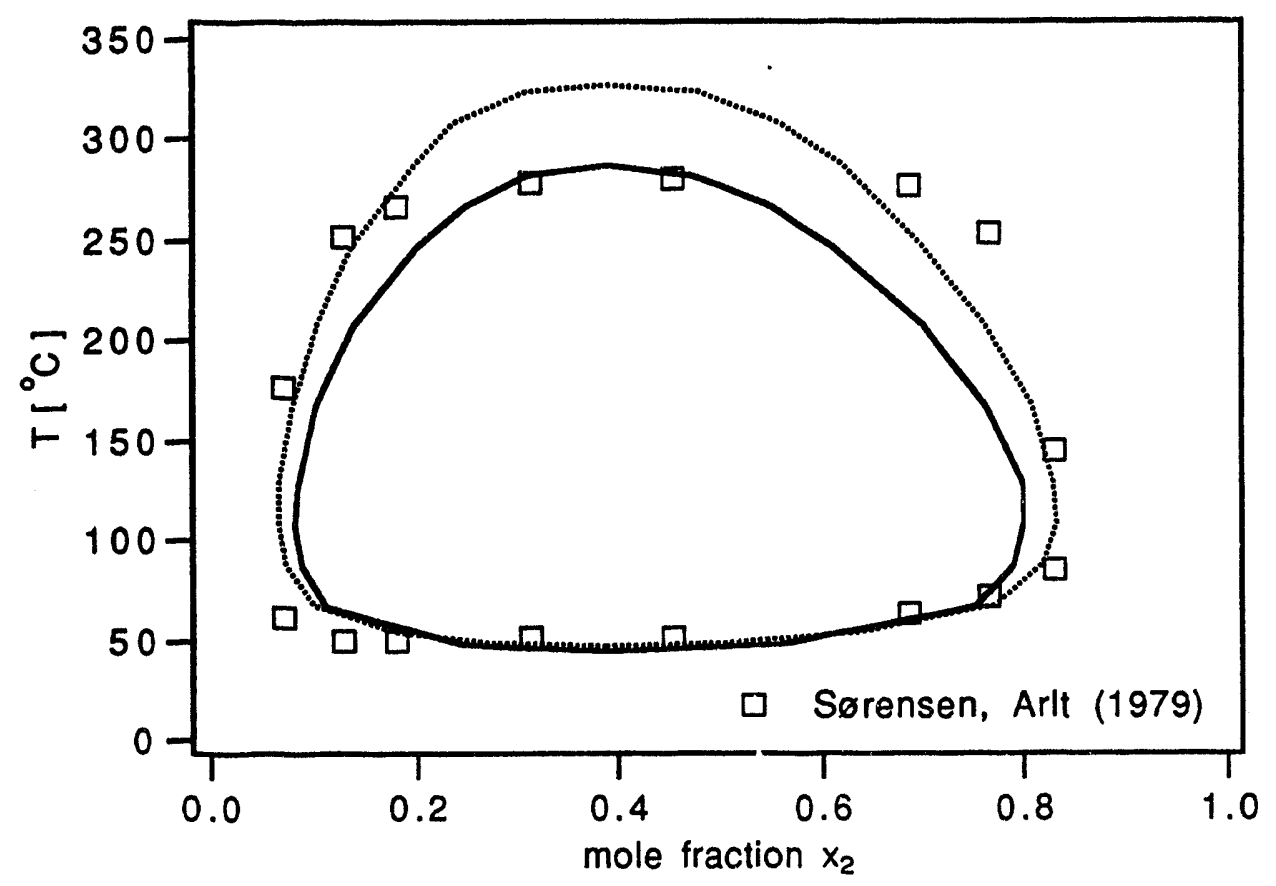

Figure 6: Closed-Loop Diagram for Glycerol (1) / Benzyl-Ethylamine (2)

Parameter: $\quad \omega=10000, \mathrm{q}_{2}=1.4$,

(solid line) $\quad \Delta \varepsilon_{12}=332[\mathrm{cal} / \mathrm{mol}], \delta \varepsilon_{12}=-5000[\mathrm{cal} / \mathrm{mol}]$

(dashed line) $\quad \Delta \varepsilon_{12}=355[\mathrm{cal} / \mathrm{mol}], \delta \varepsilon_{12}=-5100[\mathrm{cal} / \mathrm{mol}]$ 


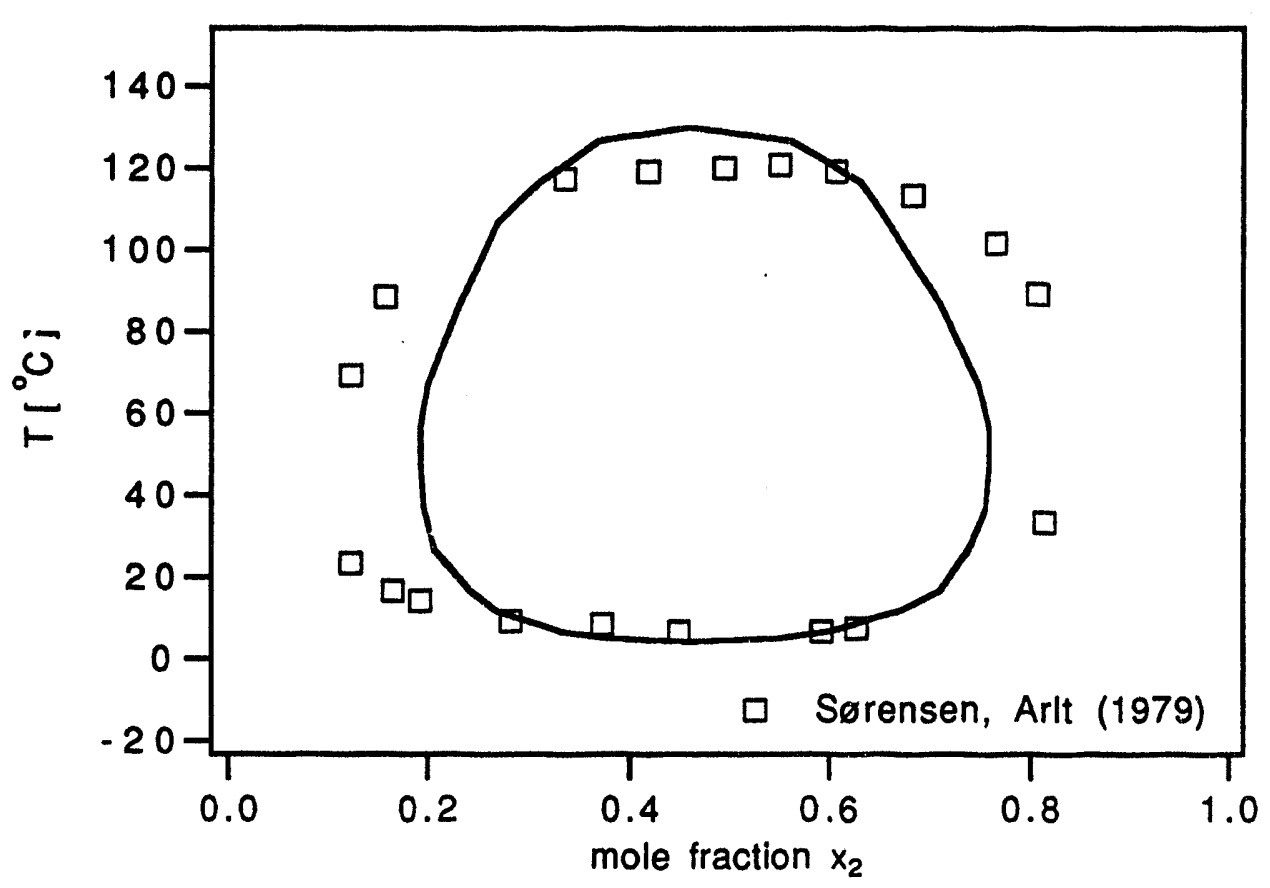

Figure 7: Cl ised-Loop Diagram for Glycerol (1)/m-Toluidine (2)

Parameter: $\Delta \varepsilon_{12}=275[\mathrm{cal} / \mathrm{mol}], \delta \varepsilon_{12}=-3900[\mathrm{cal} / \mathrm{mol}], \omega=5000, \mathrm{q}_{2}=1.1$ 


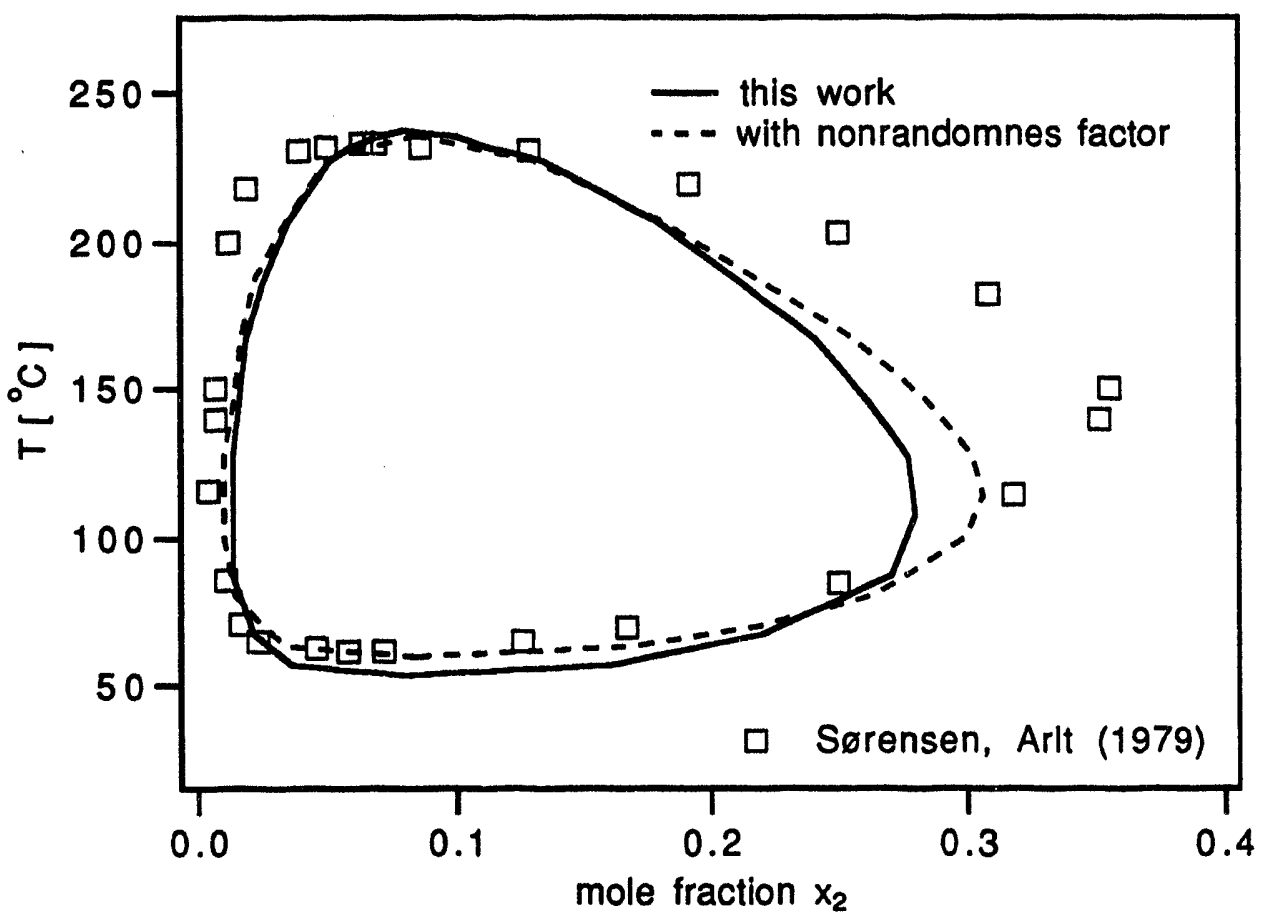

Figure 8: Closed-Loop Diagram for Water (1) / Nicotine (2)

Closed loop predicted by a nonrandom model [4] compared to a closed loop predicted by the

model, discussed in this work 


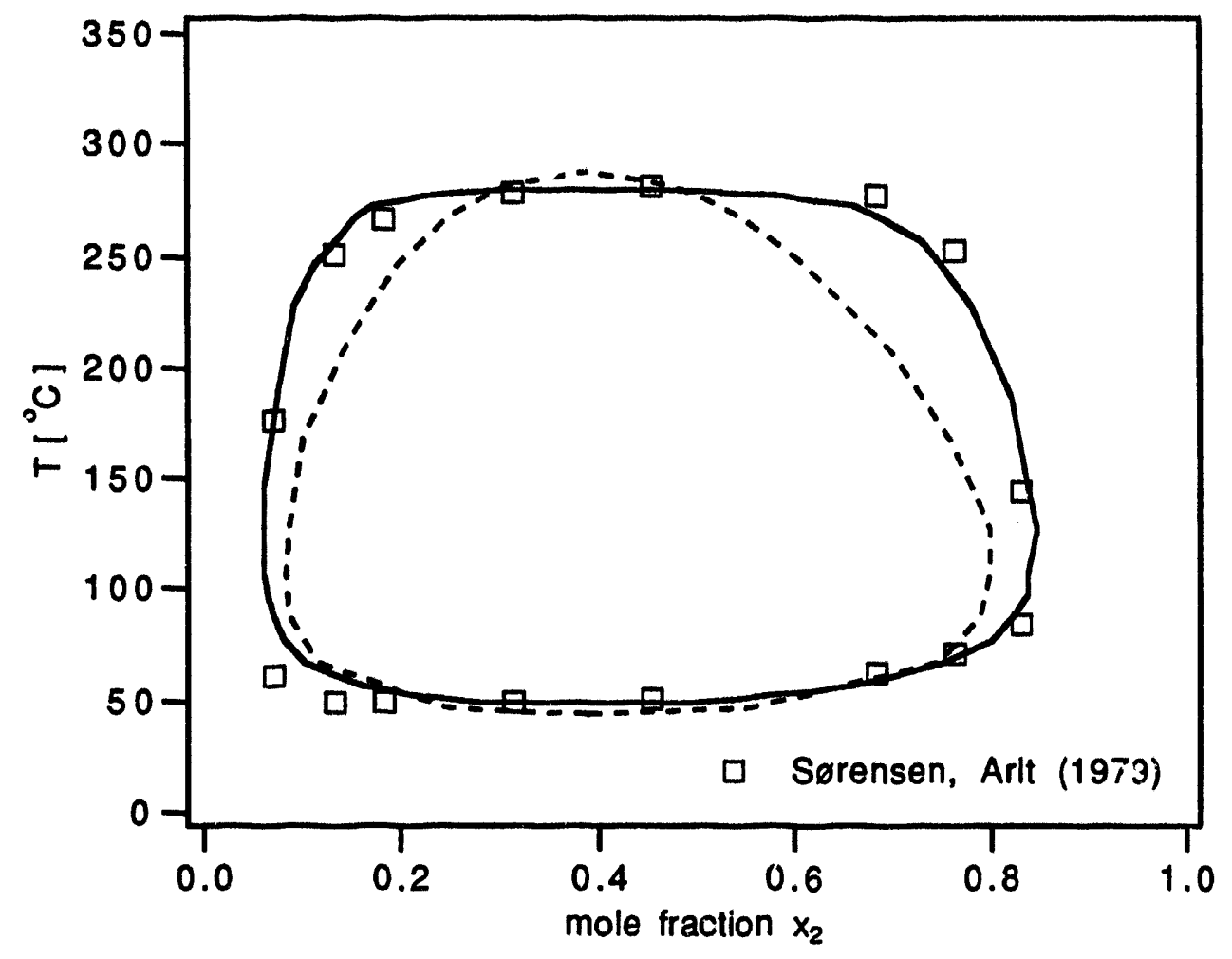

Figure 9: Closed-Loop Diagram for Glycerol (1) / Benzyl-Ethylamine (2)

broken line: van Laar-Flory Huggins model

solid line: including fluctuation corection term

Parameter: $\Delta \varepsilon_{12}=385[\mathrm{cal} / \mathrm{mol}], \delta \varepsilon_{12}=-4700[\mathrm{cal} / \mathrm{mol}] \omega=4200, \mathrm{q}_{2}=1.4$, 


\section{Notation}

$\mathrm{a}^{\mathrm{E}} \quad$ excess Helmhotz energy

B binary (temperature dependent) parameter; Eq. (2.8)

$f$ fraction of specific 1-2 interactions; Eq. (2.4)

gE excess Gibbs energy

k Boltzmann constant

$n_{i} \quad$ number of moles of component $i$

$\mathbf{N}_{\mathbf{i}}$ number of molecules of component $\mathbf{i}$

$\mathrm{N}_{\mathrm{Av}}$ Avogrado's number

$P$ pressure

$q_{i} \quad$ size parameter of component $i$

$Q_{i} \quad$ UNIQUAC surface parameter of component $i$

$R_{i} \quad$ UNIQUAC volume parameter of component $i$

$R$ general gas constant

$\mathrm{S}^{\mathrm{E}} \quad$ excess entropy

$T$ absolute temperature

$\mathrm{u}^{\mathrm{E}} \quad$ molar excess energy

UE excess energy

$v_{i}$ molar volume of component $i$

$\mathrm{V}$ volume

$x_{i} \quad$ mole fraction of component $i$

$z$ coordination number of the lattice

\section{Greek lette: $\underline{\text { s }}$}

$\alpha, \beta$ critical exponents (to describe the shape of the UCST)

$\gamma \quad$ activity coefficient

$\varepsilon \quad$ interaction energy

$\varepsilon^{\prime} \quad$ specific interaction energy

$\Delta \varepsilon_{12}=\varepsilon_{12}-0.5\left(\varepsilon_{11}+\varepsilon_{22}\right)$

$\delta \varepsilon_{12}=\varepsilon_{12}^{\prime}-\varepsilon_{12}$

$\omega$ degeneracy ratio of the Boltzmann Eq.

$\varphi_{i} \quad$ volume fraction of component $i$ 


$$
\varphi_{1}=\frac{v_{1} x_{1}}{v_{1} x_{1}+v_{2} \cdot x_{2}} \quad \text { and } \quad \varphi_{2}=\frac{v_{2} \cdot x_{2}}{v_{1} x_{1}+v_{2} \cdot x_{2}}
$$

\section{Literature}

[1] ten Brinke, G., F.K. Karasz, Lower critical Solution Temperature Behavior in Polymer Blends: Compressibility and Directional-Specific Interactions, Macromol.,17,815 (1984)

[2] J.H. Hildebrand, R.L. Scott, The Solubility of Nonelectrolytes, 3rd edition, P.139, New York: Reinhold Publishing Corporation, 1950.

[3] K. Wohl, Trans. AIChE, 42, 215 (1946).

[4] T. Hino, S.M. Lambert, D.S. Soane, J.M. Prausnitz, Lattice Thermodynamics for Binary Closed-Loop Equilibria: Orndinary and Polymer Systems, AIChE J., Vol. 39, No. 5, 837 (1993)

[5] J.J. de Pablo, J.M. Prausnitz, Thermodynamics of Liquid-Liquid Equilibria Including the Critical Region, AIChE J., 34,1595 (1988)

[6] S.C. Greer, M.R. Moldover, Thermodynamic Anomalies at Critical Points of Fluids, Ann. Rev. Phys. Chem., 32,233 (1981)

[7] M.B. Ewing,K.A. Johnson, M.L. McGlashan, The (Liquid+Liquid) Critical State of Cyclohexane and Methanol. IV: $(T, x) p$ Coexistence Curve and the Slope of the Critical Line, J. Chem. Thermody., 20(1), 49 (1988) 

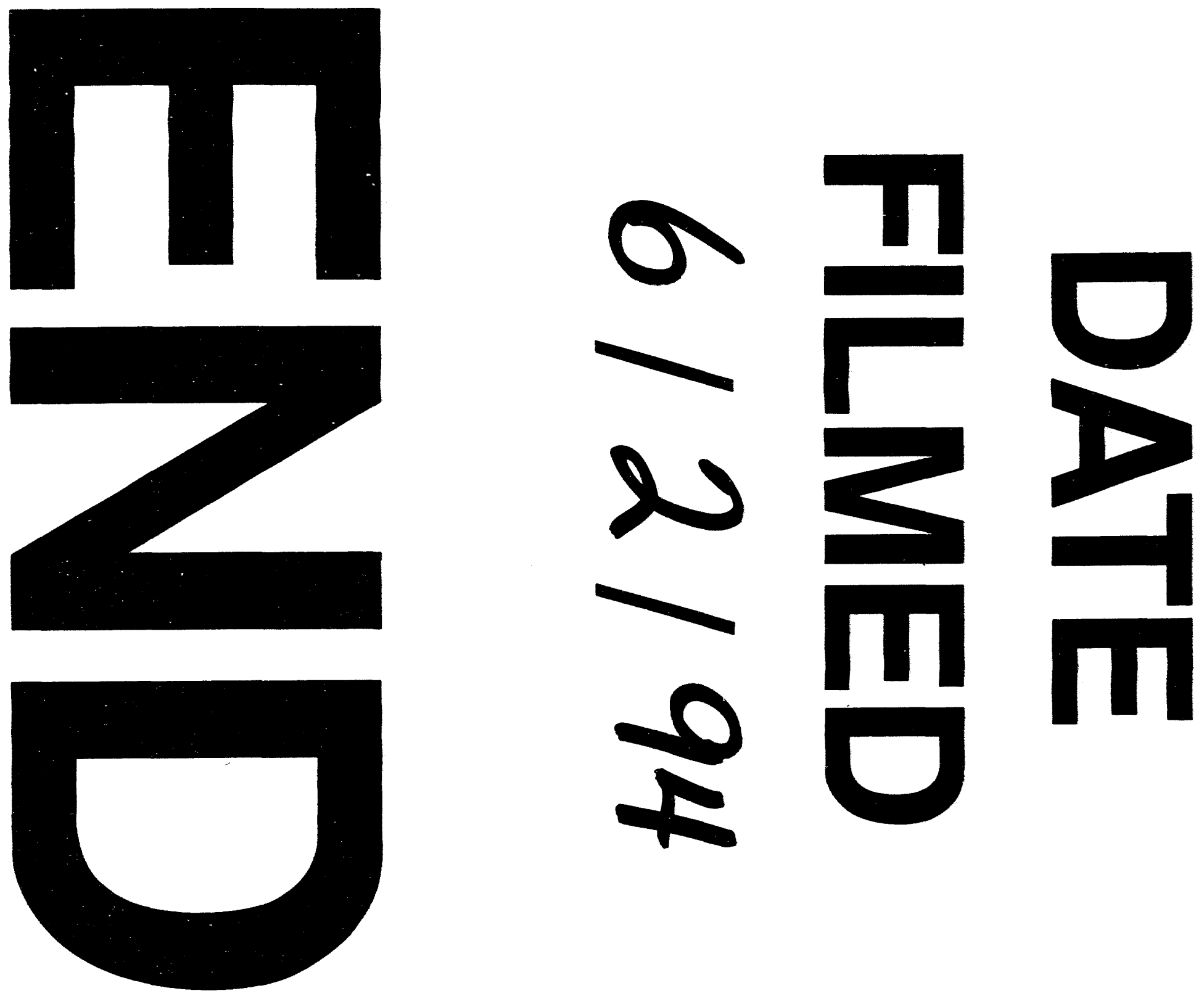
